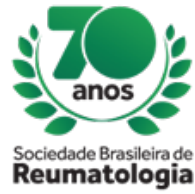

\title{
INDUCTION THERAPY WITH ORAL GLUCOCORTICOID, PULSE METHYLPREDNISOLONE OR PULSE METHYLPREDNISOLONE PLUS INTRAVENOUS IMMUNOGLOBULIN IN PATIENTS WITH SEVERE DERMATOMYOSITIS, POLYMYOSITIS AND ANTI-SYNTHETASE SYNDROME
}

Leonardo Santos Hoff (Faculdade de Medicina FMUSP, Universidade de Sao Paulo, Sao Paulo, SP, Brasil),

Samuel Katsuyuki Shinjo (Faculdade de Medicina FMUSP, Universidade de Sao Paulo, São Paulo, SP, Brasil)

\section{BACKGROUND}

Optimal treatment for systemic autoimmune myopathies (SAMs ) remains controversial. The aim of this study was to evaluate the outcomes of three different induction therapies among patients with severe SAMs.

\section{MATERIALS AND METHODS}

This retrospective single-center cohort study included consecutive adult patients with dermatomyositis, polymyositis and anti-synthetase syndrome with severe manifestations (dysphagia, bedridden muscle weakness, dyspnea secondary to interstitial lung disease, vasculitis or cutaneous rash $>30 \%$ of body surface area) from 2000 to 2017. Exclusion criteria: overlap syndromes, cancer induced myositis, followup $<6$ months not caused by death. Three main outcomes were assessed: complete clinical response [1], glucocorticoid withdrawal and mortality. Three different induction therapies were compared: oral glucocortidoid (group 1), pulse methylprednisolone (group 2) and pulse methylprednisolone plus intravenous immunoglobulin (IVIG) (group 3). Cox proportional hazards modeling and Kaplan-Meier analysis were applied to determine the differences between treatment groups.

\section{RESULTS}

132 out of 322 patients were included in the study. Group 1, group 2 and group 3 had 48, 27 and 57 patients, respectively. The following characteristics did not differ between the groups: age at diagnosis (43.0 \pm 15.6 years, $P=0.135)$, sex (102 female, 77.3\%, $P=0.233$ ), and type of SAMs -77 had dermatomyositis (58.3\%, $\mathrm{P}=0.402), 29$ had anti-synthetase syndrome (22\%, P=0.144) and 26 had polymyositis $(19.7 \%, \mathrm{P}=0.127)$. Complete clinical response was achieved in $38(79.2 \%)$ patients of group $1,24(88.9 \%)$ patients of group 2 and $46(80.7 \%)$ patients of group $3(P=0.554)$. Only $26(54.2 \%)$ patients in group 1 reached glucocorticoid withdrawal, compared to 24 (88.9\%) patients in group 2 and 41 (71.9\%) patients in group $3(\mathrm{P}=0.006)$. Mortality was not statistically different between groups: $12(25 \%)$ patients died in group 1, compared to $2(7.4 \%)$ patients in group 2 and $7(12.3 \%)$ patients in group $3(P=0.083)$. In multivariate Cox regression, treatment group 2 and treatment group 3 had a hazard ratio of $2.26(95 \% \mathrm{Cl}$ 1.19-4.29) and 2.36 (1.34-4.17) for glucocorticoid withdrawal, respectively; no association was found between the three different induction therapies and complete clinical response or mortality. KaplanMeier analysis of glucocorticoid withdrawal is shown in figure 1.

\section{CONCLUSION}

Induction therapy with pulse methylprednisolone alone or with IVIG is associated with an increased hazard ratio of glucocorticoid withdrawal in patients with severe SAMs. Complete and early corticoid suspension is a major outcome to be sought in order to diminish long term complications in patients with SAMs.

References:

1. Oddis et al.Arthritis Rheum, 2005. 